Als r96s der frühere Bundeskanzler Erhard auf dem Höhepunkt seiner Macht das Wort von der formierten Gesellschafte prägte, replizierte der heutige Wirtschaftsminister Schiller auf dem Dortmunder SPD-Parteitag I 966 mit dem Begrift der informierten Gesellschaft. Erschien dies zunächst kaum mehr als ein Wortspiel, wenn auch zugleich als Hinweis auf die Bedeutung der Information als technisches und auch pädagogisches Mitrel für die Entwicklung der Gesellschaft, so stellten die folgenden Jahre, insbesondere die Springer-Demonstrationen, die Information - die Verfügungsmacht über Informationen wie auch die Bereitschaft, sich informieren zu lassen - als das entscheidende Mittel für den Lernprozeß der Gesellschaft heraus, der dahin zielt, durch Bewußtseinsänderungen den wirtschaftlich-technischen Umbruch $\mathrm{zu}$ bewältigen und zugleich durch den Abbau von Herrschaftsstrukturen die Gesellschaft humaner und demokratischer zu gestalten.

I.

Wie wird nun die deutsche Richterschaft durch eine ihre berufliche Tärigkeit ausprechende Information auf den demokratischen Lernprozeß der Gesellschaft vorbereitet? Die Antwort wird sich aus einer Analyse der Deutschen Richterzeitung, „Organ des Deutschen Richterbundes«, ergeben, die über $80 \%$ der Richter und Staatsanwälte erreicht. Im einzelnen kann ich mich, was Bedeutung, Aufmachung und Einteilung der Zeitschrift sowie auch ihre Schrifleitung angeht, auf meine 1968 in Rasehorn-Ostermeyer-Huhn-Hasse, Im Namen des Volkes? S. 39 erschienene Analyse des Jahrgangs 1962 der DRiZ berufen. Geändert hat sich lediglich die graphische Gestaltung des Titelblatts; auch ist den Kurzbeiträgen auf Kosten der Abhandlungen mehr Raum zur Verfügung gestellt worden.

\begin{tabular}{|c|c|c|c|c|}
\hline \multirow[t]{2}{*}{ Autoren der Beiträge } & \multicolumn{2}{|c|}{ Abhandlungen } & \multicolumn{2}{|c|}{ Kurzbeiträge } \\
\hline & $\begin{array}{c}1962 \\
\% \%\end{array}$ & $\begin{array}{l}1969 \\
\%\end{array}$ & $\begin{array}{c}1962 \\
\%\end{array}$ & $\begin{array}{c}1969 \\
\%\end{array}$ \\
\hline $\begin{array}{l}\text { Establishment }{ }^{1} \\
\text { (Minister, Ministerialräte und aufwärts, } \\
\text { Richter ab Senatspräsidenten aufwärts } \\
\text { Im einzelnen: }\end{array}$ & 63 & 49 & 38 & 29 \\
\hline Minister & IO & $s$ & & \\
\hline Professoren & IO & 16 & & \\
\hline Bundesrichter & 27 & 16) & & \\
\hline $\begin{array}{l}\text { Richter der ersten Beförderungsstufe, } \\
\text { Landgerichtsdirekroren, Oberlandes- }\end{array}$ & & & & \\
\hline gerichtsräte & $\mathrm{I}_{4}$ & I9 & 13 & 29 \\
\hline Richter der Eingangsstufe und darunter & 23 & 32 & 49 & 42 \\
\hline$(\mathrm{N}=100 \%)$ & $(52)$ & (37) & $(45)$ & $\left(6_{3}\right)$ \\
\hline
\end{tabular}

I Beiträge von Rechtsanwälten sind nicht aufgeführt, da diese Gruppe in das angeführte Schema niche eingeordnet werden kann. 
Im Formalen sind Fortschritte erkennbar, die Richterschaft im ganzen stärker zur Mitgestaltung der Zeitschrift heranzuziehen.

Eindeutig kommt somit das Establishment, insbesondere bei den Abhandlungen, weniger und die untere Richterschaft mehr zu Wort. Diese Veränderung ist fast ausschließlich auf das Absinken bei den Bundesrichtern zurückzuführen, denen nahegelegt sein mag, ihre Beiträge an anderer Stelle zu veröffentlichen, wofür meine frühere Kritik Anregung gegeben hat. Es ist ferner noch auf den Verlust der Minister-Autoren im Jahre r 969 zu verweisen, dem ein gleichgroßer Gewinn der Professoren gegenüber steht.

Die stärkere Berüdksichtigung der unteren Richterschaft bei den Autoren läßt erwarten, daB die Abhandlungen auch dem Informationsinteresse des Durchschnittsrichters eher gerecht werden.

Vermutetes Interesse des Durchschnittsrichters an den Informationen auf den Seiten der Abhandlungen

\begin{tabular}{|c|c|c|}
\hline & $\begin{array}{l}1962= \\
\%\end{array}$ & $\begin{array}{l}1969 \\
\%\end{array}$ \\
\hline $\begin{array}{l}\text { starkes Interesse } \\
\text { (Justizpolitik; zum Selbstverständnis der Richter; } \\
\text { Handreichung für die Praxis) } \\
\text { überdurchschnittliches Interesse } \\
\text { (Justiz in anderen Ländern; Geschichte der Justiz; } \\
\text { Praxisspezialprobleme) }\end{array}$ & 16 & $42^{3}$ \\
\hline $\begin{array}{l}\text { Durchschnitrsinteresse } \\
\text { (fachlich Juristisches; wenig bedeutsam Justizgeschichtliches) }\end{array}$ & 57 & $2 \mathrm{I}^{3}$ \\
\hline $\begin{array}{l}\text { kein Interesse } \\
\text { (Juristische Spezialprobleme; Belangloses) }\end{array}$ & 14 & $15^{6}$ \\
\hline$(N=100 \%)$ & $(175)$ & $\overline{(135)}$ \\
\hline $\begin{array}{l}\text { In keiner anderen juristischen Zeitschrift wären } \\
\text { veröffentlicht worden }\end{array}$ & I 8 & $20^{\circ}$ \\
\hline
\end{tabular}

Unsere Erwartungen werden somit eindeurig bestärigt. Gehe ich wieder von der Annahme aus, daß ein aufgeschlossener Leser nur für die Hälfte der fachlich-juristischen Information Interesse zeigen wird, so ergibt sich ein Anstieg des Leserinteresses von $58 \%$ für 1962 auf $75 \%$ für 1969 . Demnach ist hier die früher aufgestellte Zielmarke von $85 \%$ nur um $10 \%$ unterschritten, jene $10 \%$, die sich aus Abhandlungen von Bundes- bzw. Bundesverfassungsrichtern unter Uberflüssigen zusammensetzen, ${ }^{8}$ wobei wohl die Nähe der Autoren zur Schriftleitung für die Veröffentlichung maßgebend gewesen ist.

$\mathrm{Zu}$ bedenken ist, daß wir uns bislang nur mit dem formalen Kriterium des Interesses des Durchschnittsrichters am Lesestoff der Abhandlungen befaßt

2 Belege für 1962 bei Rasehorn, Im Namen des Volkes?, S. 4I, 42.

3 Es handelt sich um folgende Beiträge: v. Münchhausen, S. 3; Ostermeyer, S. 9; Richter, S. 34 ; Ott, S. 66; Bender, S. ro5; Brüdx, S. 141; Friesenhahn, S. 169; Jansen, S. 205; Schieler, S. 207; Hamann, S. 209; Thieme, S. 237; Lisken, S. 269; Chloros, S. 27r; Neidhard, S. 316; Pulch, S. 345 ; Kübler, S. 379 ; Roxin, S. 385 .

4 Benda, S. 69; Krauss, S. 107; Bollmann, S. 142; Goldfine, S. 211; Schwande, S. 240; Wirtz, S. 305 ; Arndt, S. 311 ; Ehrig, S. 345 .

5 Lepa, S. 5; Fuhrmann, S. 13; Spranger, S. 38; Wolfram, S. 176; Drügh, S. 179; Faller, S. 184; Müller-Emmert/Friedrid, S. $273,3 \times 9,349$.

'Börtzler, S. 41; Geiger, S. 137; Amelunxen, S. 180; Vollkommer, S. 244; Kessier, S. 278.

7 Bender, S. ros; Brüds, S. 141; Amelunxen, S. 180; Hamann, S. 209; Neidhard, S. 316; Wirtz. S. 305 ; Arnde, S. 311 ; Roxin, S. 385 .

${ }^{3}$ Börtzler, S. 41 ; Geiger, S. 137 ; Kessler, S. 273. 
haben. Damit ist aber weder ein Urteil über den Wert der Information noch über die Objektivitäz der Auswahl, ob also alle Seiten und Richtungen zu Wort gekommen sind, gesprochen. Diesem Kriterium widerspricht schon, daß nur dem Establishment - 2 Professoren, je I Minister, Staatssekretär, Landgerichtspräsident - die Spalten für das wichtigste Thema, allgemeine Justiz- und Richterfragen, zur Verfügung gestellt worden sind, ${ }^{9}$ nicht aber den Justizreformern und Kritikern aus den eigenen Reihen, also Jan Berlit, Diether Huhn, Hans G. Joachim, Wolfgang Knoche, Helmut Ostermeyer, Otto Rudolf Pulch, Theo Rasehorn, Richard Schmid, Rudolf Wassermann. ${ }^{10}$ Diese einseitige Auswahl der Autoren muß den Verdacht hervorrufen, daß es weniger um die Information der Leserschaft a.ls vielmehr um die Formation der Richterschaft geht.

II.

Bevor wir diesem Verdacht nachgehen, wäre zu fragen, ob überhaupt im Rechtswesen die Bereitschaft und Fähigkeit zu unvoreingenommener Auswahl und Verwertung von Informationen besteht. Fǚr eine Analyse ist der Festvortrag von Prof. Dr. Friedrich Kübler aus Anlaß des 6ojährigen Jubiläums des Deutscien Richterbundes, Amt und Stellung des Richters in der Gesellschaft von morgen, (S. 379 f.) ein ausgezeichneter Ansatz, gerade deswegen, weil es sich um den fortschrittlichsten Beitrag des Jahrgangs handelt. So weist er klar auf die noch bestehende Befangenheit des Richters in obrigkeitlichen, "ständischbarocken " und bürgerlichen Strukturen hin, fordert darum nicht nur den demokrarischen, sondern auch den politischen Richter, weshalb er für Richterwahlausschüsse eintritt, die nur mit Parlamentariern und nicht mit Richtern besetzt sind.

Weshalb zündet dennoch nichts beim Leser? Weshalb verspürt dieser kein Zittern in den Grundfesten des Paragraphenturms? Ach - er wird ja nur mit Papier beschossen; es ist Papier-Progression. Ahnlich wie bei den großen Bildern der russischen Oktoberrevolution, die nicht die Assoziation von Revolution, sondern von Ölschinken vermitteln. Wie also der »sozialistische Realismus" kein revolutionäres Bewußtsein zu schaffen vermag, so der »juristische Akademismus « im Rechtswesen kein progressives.

Dies ist nicht nur eine Frage von Auftreten und Stil, eher noch eine solche des Informationsbezugsmusters. Korrespondierend mit der elitür hierarchischer. Stellung des Rechtslehrers im deutschen Rechtswesen wird eine elitär hierarchische Informationsauswahl von Gesellschaftsvorgängen getroffen, d. h. Selbstverständnis und Rollenidentität lassen die Annahme von "demokratischen" Informationen verweigern. So hat Kübler wohl jeden in den letzten 3 Jahren von Professoren und Assistenten geschriebenen Aufsatz über Richter- und Justizfragen gewürdigt. Was aber fehlt, ist eine Auseinandersetzung mit den Gedanken der Richter über ihren Berufsstand, also gerade mit den von Personen, die über Inside-Informationen verfügen. So mußte Kübler das besondere Phänomen übersehen, daß in den letzten Jahren eine besondere Arr der Justizkritik als Selbstkritik und nicht mehr wie vor 1933 als eine von Rechtsanwälten getragene Fremdkritik entstanden ist ${ }^{11}$, ein Vorgang, der $z$ war trotz der revolutionären

9 Friesenhahn, S. 169; Kübler, S. 379, Schieler, S. 207; v. Münchhausen, S. 3; Neidhard, S. 316.

10 Woill finden wir mit anderen Themen unter den Abhandlungen Ostermeyer und Pulch, sowie mit kürzeren Fußerungen Berlit, Huhn, Rasehorn und Wassermann.

11 Viel klarer hac dies Konrad Redeker, Unruhige Juristen - eine Zwischenbilanz, NJW I969, 1613 , geschen. 
Aufforderung hessischer Richrer, die Rosenburg - den Sitz des Bundesjustizministeriums - in Brand zu stedken ${ }^{12}$, nicht überschätzt werden darf ${ }^{13}$, mit dem sich aber eine Analyse der Justiz auseinanderzusetzen hat.

Dieser Akademismus findet empirische Untersuchungen am Objekt selbst unwesentlich wie die scholastische Medizin im Mittelalter, die die Funktionen des menschlichen Körpers nicht aus der Natur durch Sektion, sondern aus den Schriften des schon damals vor mehr als einem Jahrtausend verstorbenen Galenos ermittelte. Allerdings sind Professoren nicht bar jeder Kenntnis von Fakten. Sie kennen die veröffentlichte Rechtsprechung des Bundesgerichtshofs. Justiz erscheint hiernach letzrlich als die Institution, die dem Bundesgerichtshof die Möglichkeit gibt, Recht zu sprechen. Man sperrt sich indes gegen die Erkenntnis, daß es in $80 \%$ der Prozesse - natürlich bei der »unteren « Richterschaft - nicht um Rechts- sondern um Sozialprobleme geht, der Durchschnittsrichter somit nicht so sehr ein rechtliches, als vielmehr ein soziales Problembewuftsein haben muß. Wohin es Kübler zieht, sind die auserwählten Fälle; es muß halt eben schon - wobei er gegen mich polemisiert - ein Auschwitz- oder Contergan-Prozeß sein (S. 382) - elitäre Bezugsbilder, die Zerrbilder von der Justiz ergeben müssen. Die Reakrion von Kübler muß umsomehr Betroffenheit hervorrufen, als gerade er, bevor er die professorale Routine adaptierte, neue Wege zur Informationsverschaffung über das Bewußtsein des Richters, gerade des Durchschnittsrichters, beschritten hat, durch eine Untersuchung der DRiZ aus der Zeit von Weimarit, Wege, denen ich also nachgegangen bin.

Keineswegs soll hier ein Primat für die Untersuchungen der über InsideInformationen verfügenden Richter gefordert werden. Entscheidendes können auch - oder gerade - Nichtjuristen beitragen, die von der publizistischen Seite aus unmittelbar "am Ort « werken, wie Gerhard Mauz bei seinen SpiegelReportagen, dem die Menschlichkeit im Gerichtssaal Untersuchungsobjekt ist, oder auch der Gerechtigkeitsrigorist Ulrich Sonnemann, der unter dem leichtfertigen Umgang des Richters mit der Gerechtigkeit leiden muß. Nicht einmal auf eigene Informationen kommr es an, sondern auf den Untersuchungsansatz: das gesellschaftliche Bewußtsein des Richters. Dies zeigen die wohl bedeutendsten Justizbeiträge im Jahre 1969 , so grundverschieden sie auch sind: Wolfgang Kaupen, Die Hüter von Recht und Ordnung, und - von mir zum Teil ja heftig angegriffen - Hubert Rottleuthner, Klassenjustiz? KJ 1969, r, den wiederum Kübler nicht erwähnt, auf den auch sonst in der DRiZ nicht verwiesen wurde.

III.

Somit kann selbst ein derartig programmatisch fortschritrlicher Beitrag wie der von Kübler an den Herrschaftsstrukturen im Rechtswesen nichts ändern - die natürlich durch autoritäre Beiträge wie den von Friesenhahn (S. I59) verstärkt werden ${ }^{15}$ - ihm kommt letztilch nur eine Alibifunktion zu. Man erkennt die neuen Formierungen der DRiZ: Nicht mehr autoritäre Richtungshinweise durch Justizministerreden, sondern »rationale " Professorenerkenntnisse. Weiter: vor-

12 Vgl. Spiegel $47 / 1969,17.11 .1969$, S. 96.

$13 \mathrm{Vgl}$. Theo Rasehorn, Von der Klassenjustiz zum Ende der Justiz, Krit. Just. I969, S. 273; Hubert Rottleuthner, Klassenjustiz?, Krit. Just. I969, S. I.

14 Der Deutsche Richter und das demokratische Gesetz, AcP I62 (1963) S. I04 ff.

15 Vgl. meine Auseinandersetzung mit Friesenhahn in: Opas Justiz lebr, bei Ulrich Sonnemann. Wie frei ist unsere Justiz, 1969 , S. 13 ff. 
sichtiges Taktieren gegenüber politischen Erfordernissen; - unter der Dedke aber die alten autoritären Strukturen.

Verfolgen wir diese These bei der Untersuchung der Darstellung aktueller Justizprobleme aus dem Jahre 1969:

\section{a) Demonstrationsrecht}

Vorsichtiges Taktieren kommt als erster Eindruck auf, wenn die Darstellung der Demonstrationsprobleme unter die Lupe genommen wird. Wenn ihnen auch ein ganzes Heft - Märzheft - eingeräumt worden ist, so muß gleichwohl die Behandlung dieser von der Offentlichkeit in den Jahren I $968 / 69$ als vorrangig angesehenen Rechtsprobleme als oberfächlich bezeichnet werden. Kein Richter wurde mit einer umfassenden Darstellung der Problematik betraut. Unter den Abhandlungen befinder sich kein Originalbeitrag. Referiert wird aus einem Polizeikommentar zum Versammlungsgesetz; es folgen Reden, eine des fortschrittlichen Sieghart Ott und als Gegenstück eine vom Bundesinnenminister Benda zum Demonstrationsrecht. Auch unter den Kurzbeiträgen gibt es Auszüge von an anderen Stellen erschienenen Aufsätzen. Lediglich s Beiträge mit insgesamt etwa $5^{1 / 2}$ Seiten sind Original. $Z_{\text {wei Beiträge der Reformer Berlit und }}$ Wassermann über Verhalten bei Demonstrationsprozessen erscheinen relativ autoritär, wohl eine Reaktion auf die Apo-Gerichtskrawalle im Winter 1968/69. Drei weitere befassen sich mit Einzelfragen, wobei die von Sandweg und Hahnzog (S. 73, 74) eine leicht fortschrittliche Tendenz haben. Der längste, fast 2 Seiten lange Beitrag, eine rechtsvergleichende Studie über das schwedische Recht, stärkt autoritären Richtern das Rückgrat: $\gg$ Das Beispiel Schwedens, eines Staates mit einer alten Freiheitstradition, zeigt, daß sich freiheitliche Grundrechte und ein energischer Schutz der öffentlichen Ordnung nicht ausschließen « (Schmidt, S. 78).

Vorsichtiges Taktieren also nach außen; unter der Decke aber die autoritären Strukturen. So teilte die Schriftleitung unter $\gg$ Leserzuschriften « mit (S. 86), sie habe einen Universitätsprofessor um einen Beitrag zum Demonstrationsrecht gebeten (Natürlich wieder ein Professor!), der den Auftrag wegen starker Belastung abgelehnt aber ihr wie folgt geschrieben habe:

"Ich finde es traurig, daß über dieses Thema heute geschrieben werden muß. Ich halte derartige Rechtfertigungen bei an sich strafbarem Demonstrationsverlauf für grotesk, verfassungswidrig und mi: der bestehenden Rechtsstaatennorm völlig unvereinbar. Auf diesem Gebiet wird mit derartig unsinnigen Argumenten gearbeitet, daß es in der Tat rotwendig erscheint, die an sich einfache Rechrslage mir allem Nachdruck darzustellen...."

Während einmal mehr die umfassende Rechtsprechungsïbersicht als informierend und objektiv ausgewählt zu loben ist ${ }^{10}$, steht es ganz anders mit der Presseschatt. Wird sie danach ausgewertet, ob bei den Demonstrationen im wesentlichen positive oder negative Aspekte überwiegen, so unterstreichen alle II $\mathrm{Zi}$ tate eher die negative Seite. Etwas objektiver ist die Auswahl der Pressestimmen zu den Demonstrationsprozessen. Bei 3 Zitaten wird bezweifelt, ob sich Richter gegenüber Demonstranten richtig verhalten haben, bei 8 wird es bejaht. Die eigene autoritäre Einstellung der Schrifleitung zeigt sich bei einer eigenen

16 Vgl. auch meinen Hinweis in: Im Namen des Volkes, S. †०. 
Stellungnahme zum Marburger Fall einer Verhöhnung des Gerichts durch Auftreten eines Angeklagten in Robe:

^Bei so groben Störungen durch böswillige Elemente, wie wir sie in letzter Zeit erlebt haben, reicht als Ordnungsmaßnahme eine Strafe von höchstens 3 Tagen nicht mehr aus $\alpha$. (S. 15s)

\section{b) Vorbengehaft}

Die Anfang Ig69 aktuell gewordene Frage der Vorbeugehaft wird nur knapp behandelt, insbesondere durch zwei Berichte über eine Gesamtvorstandssitzung des Deurschen Richterbundes (S. I00, 13I). Der geschäftsführende Vorstand fand mit seiner Ablehnung des CDU-SPD-Entwurfs bei dem erweiterten Vorstand keine Mehrheit. Dieser wollte zwar gewisse Modifikationen, auch einen anderen Namen - Sicherungshaft -, aber keine materialen Änderungen. Vorgezeichnet war die Stellungnahme der Mehrheit dieses Mal durch einen eigenen Beitrag in der DRiZ, eines Staatsanwalts, über die Legitimitït der Sicherungshaft, mit dem der Gesetzesentwurf begrüßr wurde, »um den bisher geduldeten Etiketrenschwindel zu beseitigen und Klarheit zu schaffer" " (Spranger, S. 38).

\section{c) Parlamentarischer Richterwablausschuß}

Diese Frage wurde 1969 justizintern leidenschaftlich diskutiert, ohne daß die Offentlichkeit daran teilnahm. Es geht um Ausschüsse, gebildet von Parlamentariern und von der Richterschaft gewählten Richtern, die statt des Justizministers über Ennennung und Beförderung der Richter befinden. Sie bestehen bereits, zumeist aber ohne Beförderungsbefugnis, in Hessen, Berlin, Hamburg und Bremen. Die Sache bekam einen lebhaften Auftrieb, als in NordrheinWestfalen die in der Opposition stehende CDU einen Gesetzesentwurf für einen derartigen Ausschuß einbrachte.

Hier nahm die DRiZ of fiziell Partei. Sie unterstiutzte den CDU-Antrag mit zwei engagiert geschriebenen Aufsätzen, mit denen jeweils sogar ein Monatsheft eingeleitet wurde (Jansen, S. 205; Lisken, S. 269). Geht man tiefer, so muß auch bier der Schriftleitung ein vorsichtiges Taktieren nachgesagt werden, handelt es sich doch einmal um Konzessionen an eine Gruppe im DRB-Vorstand, der die beiden Verfasser nahestanden, Konzessionen aber auch an die Parteien selbst, die sich immer stärker für derartige Ausschüsse einsetzen.

$\mathrm{Da}$ es sich nur um Lippenbekenntrisse der Schriftleitung handelte, daß man auch hier von den autoritären Strukturen nicht loskommt, zeigen die Stellungnahmen unter dem Strich; denn hier gibt es, sieht man von dem Aufsatz von Uhlig ab, der zur Grundproblematik keine Stellung nimmt (S. 357), nur ablehnende Stimmen, mit dem stärksten Nachdruck aus Bayern (S. 26I, 299, 302, 322, 371). Für die konservativen Kräfte geht es ja hierbei nicht nur um eine Frage der Weltanschauung, sondern um eine der Existenz. Durch die Wahlausschüsse könnte nämlich ein gewisser politischer und fortschrittlicher Richtertyp nach vorn kommen, wofür es beim Bundesverfassungsgericht schon gewisse Ansätze gibt. Die Chance dazu ist allerdings sehr gering, was sich darin zeigt, $\mathrm{da} B$ bislang beim BGH vorwiegend konservative "Superjuristen " gewählt worden sind, da hier mit dem Vorschlagsrecht der Justizbürokratie die personell nicht informierten Parlamentarier überspielt werden konnten. 
Wenn also diese Ausschüsse schwerlich den Erfolg haben werden, den gerade Kübler erwartet (S. 384), so mußte dennoch die bloße Möglichkeit einer Umfunktionierung des Richtertyps konservative Kräfte erschrecken. Sie warnen vor einem Eindringen von "parteipolitischem Opportunismus in die Richterschaft und von einer $\gg$ Abkehr vom Leistungsprinzip" (S. 261, 299, 302, 322, $37 \mathrm{r})$. Es ist bezeichnend, daß es sich die beiden Befürworter nur erlauben können, sehr versteckt von einer anderen, politischen, Ausrichtung der Richterschaft zu sprechen, sie argumentieren zumeist verfassungsrechtlich: strenge Trennung der Gewalten, Verwirklichung der vom Grundgesetz geforderten Stellung des Richters, oder auch mit allgemeinen Erwägungen wie Stärkung des Richterprestiges und eine "gerechtere Auswahl« der Richter.

\section{d) Neue Formen im Gerichtssaal}

Bei diesem in der Offentlichkeit lebhaft diskutierten Problem, wobei dem Frankfurter Symposium im April 1969 zwischen Richtern, Staatsanwälten und Journalisten - über das zwei Teilnehmer B(erlit) und W(assermann) gemeinsam referierten (S. 250) - eine besondere Bedeutung zukam, hat die DRiZ im besonderen Maße konservativen Kräften Raum gegeben. Dabei mag von Bedeutung gewesen sein, daß hier - anders als bei der Frage der Wahlausschüsse - sich die politische Offentlichkeit - Justizministerien und Parteien - nur zögernd für Reformen aussprachen, die bislang im Wegfall der schwarzen Bäckermütze, des Baretts, gipfelten (S. 326, Anm. ro).

Im übrigen fand die Schriftleitung auch dieses Thema nicht widutig genug, um es in einer Abhandlung ausführlich zu erläutern, insbesondere von den Befürwortern einer Reform erläutern zu lassen. Die Meinung der Reformer, sieht man von dem objektiven Tagungsbericht selbst ab, kommt überhaupt nicht zu Gehör. Alle wertenden Außerungen - wird von einem Leserbrief eines jungen Richters, wohl eines starken Rauchers, abgesehen, der dem Angeklagten die RaucherWaffengleichheit einräumen möchte $\left(S_{3} 3_{3}\right)$ - wenden sich gegen neue Formen, halten Roben für demokratisch (v. Schlotheim S. 325 , v. Lanzenauer, S. 283 , Klein S. 288) oder heben die Praktabilität der bisherigen Formen hervor (Salger S. 357, v. Scthotheim S. 325), wobei noch weiter argumentiert wird, letzrlich komme es auch auf die Würde des Richters, nicht nur auf die des Angeklagten an, dem es ja nicht um Auseinandersetzung mit dem Ridhter, um Recht und Wahrheit, sondern nur um den eigenen Vorteil gehe (Klein S. 288).

Natürlich ist es, vom Grundsätzlichen her gesehen, nur von geringer Bedeutung, wie Redeker betont ${ }^{17}$, wie die Sitzungssäle ausgestaltet sind und ob mit oder ohne Robe judiziert wird (So auch Prüfer, S. 362). Wenn aber autoritäre Kräfte diesen Formalien, wie angeführt, eine derartige Bedeutung beimessen, so muß gerade darum hier die Chance der Konfrontation genutzt werden ${ }^{19}$.

\section{e) Rebseurteil und richterliches Selbstvcrständnis}

Zeigre sich 1969 die Zeitschrift anders als 1962 nach außen eher behursam, wenn auch das autoritäre Grundmuster unter dem Strich erkennbar blieb, so ist indes ein Vorgang herauszuheben, bei dem - man möchte sagen: - der alte Adam

$17 \mathrm{Vgl}$. Anm. Ir.

18 So Rudolf Wassermann, Neuer Stil im StrafprozcB, ZRP I969, 169 (172). 
durchbrach: Die Reaktion auf den Freispruch des ehemaligen Richters an NS-

Volksgerichtshof Rehse durch ein Berliner Schwurgericht im Dezember 1968.

Zunächst ist auch hier festzuhalten, daß das Rechtsproblem selbst - die gerade doch für Richter entscheidenden Fragen wie Rechtsbeugung und Richterprivileg höchst oberflächlich auf $2 / 3$ Seiten ohne Anführung der maßgeblichen Literatur behandelt wurde (Steffen S. 45). ${ }^{19}$ Zum Urteil selbst hat die DRiZ eine Auswahl von Ix Kommentaren sowie Stimmen aus der Presse und den Richterverbänden gebracht, von denen nur 3 das Urteil angriffen, die übrigen es verteidigten oder "abwägender " Natur waren. Noch krasser ist das Verhältnis bei Zugrundelegung der Seitenzahl der gesamten Veröffentlichungen: Eine halbe Seite für die Kritiker; fast 3 Seiten für die Gegenpartei. Wer sich die damalige Reaktion in der Offentlichkeit vergegenwärtigt, das Aufbäumen gegen den Freispruch, muß schon das Findergliick der Schriftleitung bewundern, eine derartige Anzahl entgegengesetzter Auffassungen überhaupt zu entdecken.

Wer sich nur an Hand der DRiZ über das Rehse-Urteil informiert hätte, dem mußte sich der Schluß aufdrängen, es habe sich vorwiegend die mniedrige Journalistik «̈̈ber das Urteil erregt, besser Informierte hätten aber erkannt, $\mathrm{da} B$ die Berliner Richter nicht anders hätten entscheiden können, ja, daß das von Richtern für Richter aus $\$ 336$ SrGB geschaffene Privileg »ein Schutzwall sei, den die Rechtsgemeinschaft zu ihrer Befriedigung bedarf " (Steffen S. 45).

Hier zeigt sich somit evident ein autoritäres und damit eben ein zurückgebliebenes gesellschaftliches Bewußtsein. Der Beitrag der Richterschaft an der öffentlichen Diskussion um Rehse und das Urteil hätte doch gerade in einer umfassenden und rückhaltlosen Klarlegung der verschiedenen Standpunkte liegen müssen. Gerade von den Richtern als den Fachkennern erwartete die Gesellschaft, daß sie sich von niemanden in der Kritik übertreffen ließen. Betrieben wurde aber gerade ein Absichern gegenüber der Gesellschaft und ein Formieren der eigenen Reihen.

Aber letztlich ist sie nicht einmal so sehr beängstigend als vielmehr erbarmenswert: Opas Justiz. - Und so feiert sie sich auch. Mit jenem abgedroschenen Pathos, der heute nur noch zu Jubelfeiern von Dorfgesangsvereinen paßt, wird der 60 Jahre Deutscher Richterbund und seiner Vorsitzenden gedacht. Da liest man über den ersten Vorsitzenden Leeb: „Dabei sah es zunächst gar nicht so aus, als ob er, der Bauernbub... dereinst ein Führer der deutschen Richter werden würde.« (H. Arndt, S. 3I2). Man sollte etwas gegen Männer haben, die, ob sie Bauern- oder Zöllnerbub gewesen sind, später Führer von irgendwas werden! $\mathrm{Daß}$ Leeb mit diesem Vergleich kein Unrecht geschieht, zeigt die an gleicher Stelle wiedergegebene kurze Blütenlese aus seinen Aufsätzen zwischen rgrg bis I922: "Was sich Rechtsstaat nennt, ist ein Staat ohne Recht...»» Wo mehrere Parteien die Herrschaft ausüben, entstehen Kompromißgesetze. Sie stellen Mischlinge, Kreuzungen der Belange der herrschenden Parteien, Bastardrecht dar.» Für Arndt ist dennoch Leeb, dem selbst der gemäßigte Kübler ein »antidemokratisches Ressentiment « attestiert ${ }^{20}$, ein »unerschrodsener Kämpfer für Richter und Recht«. Noch großartiger wird er und der Deutsche Richterbund in dem Festaufsatz von Wirtz gefeiert (S. 306):

19 Man vergleiche dagegen wie gründlich die Anwalsszeirschrifta, die NJW, diesen Fragen nachgegangen ist. Belege bei Theo Rasehorn, Das Verfahren gegen Rehse und die Problemarik des $\$ 336$ StGB, NJW 1969,457 .

20 A. a. O., S. 113 (Anm. 14). 
$\gg$ Der DRB kann... innerlich gefestigt die Kriegszeit der Jahre r9r4 bis rgrs überstehen und aus der ersten großen Bewährungsprobe im November-Umsturz I 918 geläutcrt und gestärkt hervorgchen ... Er hatte hierbei das Glüdk, an seiner Spitze noch den ersten Bundesvorsitzenden Dr. Leeb zu wissen, der das Schiff der Getreuen behutsam und mutig zu geuen Ufern steuert.*

Was von der »Läuterung «, „Behutsamkeit « und »den neuen Ufern « zu halten ist, das hat Kübler, a. a. O., zwar zurückhaltend, aber doch deutlich aufgezeigt. Die Geschichtsklitterung erreicht bei Wirtz noch einen weiteren Höhepunkt. Zu "neuen Ufern" versuchten nach dem ersten Weltirieg tatsächlich demokratische Richter aufzubrechen, die darum auch in dem "Schiff der Getreuen « keinen Platz mehr fanden und ihren eigenen Bund gründeten und deshalb vom DRB heftig befehdet wurden. Jetzt aber werden sie wie folgt vereinnahmt: "Dem DRB erwächst... die zusätzliche Aufgabe, sich im Kampf mit dem Republikanischen Richterbund schützend vor seine Mitylieder zu stellen « (S. 307).21

IV.

Fassen wir zusammen: der Jahrgang 1969 spiegelt nicht mehr wie der von 1962 die heile Welt wieder. Darum erfolgen auch keine Aufrufe nach draußen zur Umkehr und Besinnung mehr. Aber man will auch nicht die Welt in die Gerichtssäle hereinlassen, wie dies progressive Richter vorschlagen, man kapselt sich ab, weicht Auseinandersetzungen aus. Heiße Probleme wie Demonstrationsdelikte, Fall Rehse, neue Formen im Gerichtssaal werden nur aus zweiter Hand behandelt, dafür aber unter dem Strich - Leserbriefe, Kommentare, Presseauswahl deutliche Hinweise gegeben, wie sich ein »guter« Richter zu diesen Fragen zu stellen hat. Richterliche Justizkritiker werden an den Rand gedrängt. »Wie ich sehe, haben Sie Gelegenheit, an anderer Stelle zu publizieren«, kann die Ablehnung eines Aufsatzes lauten.

Allerdings temüht sich die Schriftleitung stärker um die Resonanz in den eigenen Reihen. Statt autoritärer Richtungsanweisung wie r 962 vergewissert man sich jetzt stärker der Zustimmung der Mehrheit der Richter, was bei deren konservativer Grundeinstellung ein leichtes Unterfangen ist. Diese Mehrheit wird zur Elite stilisiert. Sie ist darum nicht mehr Gefolgschaft bei Justizministerreden, sondern darf sich mit Professoren gemeinsam dem Recht verpllichtet wissen. Allerdings erfährt der Leser kaum erwas über die echten Auseinandersetzungen $z$ wischen Justiz und Gesellschaft, insoweit muß er sich durch den „Spiegel« oder die »Zeit«, durch die »Vorgänge«, »Recht und Politik« oder durch diese Zeitschrift informieren lassen.

So bleibt letztlich das mediokre Provinzielle eines Vereinsblatts. Aber gerade eine solche Haltung fördert das Bedürfnis nach Leitung und Führung wie ja auch unser provinziellster Politiker, Ludwig Erhard, der Sehnsucht nach der formierten Gesellschaft Ausdruck gegeben hat. Je vreniger informiert wird, um so besser kann ja formiert werden. Dennoch scheinen einer demokratischen Gesellschaft von der Richterschaft aus zur Zeit keine großen Gefahren zu drohen, dafür ist deren Instrumentarium noch zu sehr veraltet und zu wenig managerial. Wirtz gibt im Schlußwort seines Jubliäumsaufsatzes den Richtern folgenden futurologischen Hinweis, wie sie die Zukuntt bestehen sollen:

Frischauf zu weiteren Taten!

Ach ja...

Theo Raseborn

21 Der Protestbrief von Diether Huhn ist allerdings veröfientiche worden, S. 396. 